\title{
The Meaning of Verb Falloir and other Impersonal Verbs in French and Its Equivalences in Indonesian Language
}

\author{
Balduin Pakpahan ${ }^{1}$, Pengadilen Sembiring ${ }^{2}$, Nurilam Harianja ${ }^{3}$,Angel Marni ${ }^{4}$ \\ \{Balduinpakpahan113@gmail.com\} \\ Fakultas Bahasa dan Seni, Universitas Negeri Medan, Indonesia ${ }^{1,2,3}$
}

\begin{abstract}
This study aimed to explain the meaning of Verb especially Falloir in French and Its Equivalence in Indonesian Language. Impersonal verb is a verb where its subject is abstract (sujet apparent). However, in fact, the study result showed that the abstract subject could also appear in its indonesian language translation. Its apperance was made coherent with its indonesian language structure which is sentence context. While in some cases, if impersonal verbs are followed by a nominal or equivalent group, it represents the real subject which has an intermediate status between the subject and the complement. Based on the study result found that impersonal verb falloir could be equivalent in French with other impersonal verbs such as il est necessaire, il est oblige, il est éxasperant, and other verbs in the forms of imperative and devoir. Meanwhile, in Indonesian language, literal meaning of il faut "harus" have synonymous forms such as mesti (semestinya), perlu, wajib, and patut. If all expressions of equivalence of il faut were made into negative forms, the sentence could have antonymous meaning. The data sources used were 2 novels written in french and their translation, and 2 novels written in Indonesian and their translation. This study used equivalence method by making Indonesian language as its determination tool.
\end{abstract}

Keywords: Verbs impersonals, Meaning, Equivalence Word, French, Indonesian Language

\section{Introduction}

French expresses tense(temps), aspect(aspect), and modus(mode) in a verb through its interpretation form. Meanwhile in Indonesian language, there is no tense verb(temps du verbe) and grammatical aspect use is facultative. Hoed (1989) said that in Indonesian language, perspective aspect equivalence (passé composé atau passé simple) is sudah (perfect tense),telah (perfect tense), or action way. So, in Indonesian language, there is no morphological change on the verb form to determine an aspect of a sentence. Samsuri (1981: 252) added that Indonesian language does not use morphological change to state an aspect, but particles telah/sudah (past simple), sedang/lagi (present progressive), and akan (future tense) that are not always found in sentence.

Basically, in verb construction, there is a sentence meaning expression which is usually explicitly. So, to understand the sentence meaning, it is needed a strong reasoning on the verb interpretation form. Because French is a less of special morphological signs as time sign and aspect of a sentence. This is equivalent what Lachet (2013) said, en ce qui concerne l'aspect 
grammatical, le français est une langue qui est dépourvue de marquage morphologique spécifique.

This paper is going to explain how impersonal verb falloir which has meaning harus (must) can be equivalent with other impersonal verb in French. Its equivalence in Indonesian language if seen from its literal meaning does not have a correlation at all with falloir. This shows how implicit meaning of the verb is said in French and Indonesian language. The study result would be very important for students and teachers of French who would like to closer understand the use of impersonal verb, especially falloir.

This research was done with equivalence method where Indonesian language as the reference. The study resources are 2 French novels and their translation and two Indonesian novels and their translation.

Equivalence method in this research included basic technic and continuence technic. The basic technic used is Pilah Unsur Penentu (PUP). PUP is used to classify grammatical meaning of verb conjunction through time, aspect, and modus whichcan affect to lexical meaning in French and Indonesian language. Next, continuence technic used is Hubung Banding Menyamakan (HBM) which is relevant with its determined data (Sudaryanto, 1993:27).

In the next part would be showing datas of impersonal verb falloir and its meaning harus and its equivalence in French and Indonesian language which was implicitly expressed.

\section{Discussion}

Table 1: Apperance list of verb Falloir in French and its interpretation on Les Mots and its interpretation in Indonesian language words.

\begin{tabular}{|l|l|l|}
\hline \multirow{2}{*}{ No. } & \multicolumn{3}{|c|}{ FALLOIR } \\
\cline { 2 - 4 } & French & Indonesian \\
\hline 1. & $\begin{array}{l}\text { Il ne faut pas même remuer } \\
\text { l'orteil ; }\end{array}$ & Jempolkakipuntidakkugerakkan \\
\hline 2. & $\begin{array}{l}\text { Je me lançai dans une incroyable } \\
\text { aventure; il fallait grimper sur } \\
\text { les chaises, sur les tables.. }\end{array}$ & $\begin{array}{l}\text { Akuterjundalampetualangan } \\
\text { yang luarbiasa; menaikikursi, } \\
\text { meja .... }\end{array}$ \\
\hline 3. & $\begin{array}{l}\text { Cette absence prodigieuse le } \\
\text { transfigura. Il s'en fallait de } \\
\text { beaucoup que l'Institut fût au } \\
\text { complet; }\end{array}$ & $\begin{array}{l}\text { Ketidakhadiranitumenyulapnya. } \\
\text { MemangtidaksemuaanggotaInstit } \\
\text { uthadir }\end{array}$ \\
\hline
\end{tabular}

Table 2: Apperance list of verb Falloir in French and its interpretation in Indonesian language on Thérèse Raquin and its interpretation Thérèse Raquin

\begin{tabular}{|l|l|l|}
\hline \multirow{2}{*}{ No. } & \multicolumn{2}{|c|}{ FALLOIR } \\
\cline { 2 - 3 } & French & Indonesian \\
\hline 1. & $\begin{array}{l}\text { Elle demeurait affaissée sur } \\
\text { sa chaise, }\end{array}$ & Iaharusdudukterenyak di kursinya. \\
\hline 2. & $\begin{array}{l}\text { Tu sais, lui dit-il, il faut que je } \\
\text { fasse ton potrait. }\end{array}$ & Akuakanmenulispotretmu. \\
\hline
\end{tabular}




\begin{tabular}{|l|l|l|}
\hline 3. & $\begin{array}{l}\text { D'autre part, depuis } \\
\text { longtemps, il n'avait pas } \\
\text { contenté ses appétits; Il } \\
\text { sevrait sa chair, et il ne } \\
\text { voulait point laisser échapper } \\
\text { l'occasion de la repaître un } \\
\text { peu. }\end{array}$ & $\begin{array}{l}\text { Sementaraitu, sudah lama sekali ia } \\
\text { tidak memuaskan hasratnya; } \\
\text { uangnya tidak banyak, ia harus } \\
\text { memendam keinginan tubuhnya dan } \\
\text { tak mau kehilangan kesempatan } \\
\text { untuk menikmatinya sedikit. }\end{array}$ \\
\hline 4. & $\begin{array}{l}\text { Dans la boutique, sa } \\
\text { maîtresse devenait une femme } \\
\text { comme une autre, qu'il ne } \\
\text { fallait point embrasser et qui } \\
\text { n'existait pas pour lui. }\end{array}$ & $\begin{array}{l}\text { Di dalam toko, wanita } \\
\text { selingkuhannya adalah wanita biasa, } \\
\text { sama dengan wanita-wanita lainnya, } \\
\text { yang mungkin tidak akan diciumnya, } \\
\text { dan kehadirannya tidak akan berarti } \\
\text { apa-apa baginya. }\end{array}$ \\
\hline 5. & $\begin{array}{l}\text { J'ai besoin de croire que la } \\
\text { police est bien faite }\end{array}$ & $\begin{array}{l}\text { Aku harus percaya bahwa } \\
\text { kepolisian bekerja dengan baik }\end{array}$ \\
\hline 6. & $\begin{array}{l}\text { Diable, dit-il, il ne va pas } \\
\text { falloir remuer là-dedans. }\end{array}$ & $\begin{array}{l}\text { "Sialan," katanya. Kita lebih baik } \\
\text { tidak bergerak-gerak dalam perahu } \\
\text { ini. }\end{array}$ \\
\hline 7. & $\begin{array}{l}\text { Elle tendait ses volontés de } \\
\text { toutes ses forces, car elle } \\
\text { avait peur d'éclater en } \\
\text { sanglots et de tomber à terre. }\end{array}$ & $\begin{array}{l}\text { Ia harus mengerahkan seluruh } \\
\text { kekuatannya untuk mengendalikan } \\
\text { diri, karna ia takut akan menangis } \\
\text { tersedu-sedu dan tercebur kedalam } \\
\text { air. }\end{array}$ \\
\hline 8. & $\begin{array}{l}\text { C'est ma faute, cria-t-il, je } \\
\text { n'aurais pas dû laisser ce } \\
\text { pauvre garçon danser et } \\
\text { remuer comme il le faisait... }\end{array}$ & $\begin{array}{l}\text { "Ini salahku, tangisnya." Aku } \\
\text { semestinya tidak membiarkan pria } \\
\text { malang itu menari-nari dan } \\
\text { mengguncangkan perahu.... }\end{array}$ \\
\hline
\end{tabular}

Table 3: Apperance list of verb Falloir in French as lexes in Indonesian language in the novel "Keberangkatan" and its equivalence in French in the novel Le Départ

\begin{tabular}{|c|c|c|}
\hline \multirow{2}{*}{ No. } & \multicolumn{2}{|c|}{ FALLOIR } \\
\hline & Indonesian & French \\
\hline 1. & Itu juga tidak baik. & $\begin{array}{l}\text { Mais ce n'est pas ce qu'il faut faire } \\
\text { non plus. }\end{array}$ \\
\hline 2. & $\begin{array}{l}\text { Tetapi lebih sering kami } \\
\text { menunggu hingga tiba giliran. }\end{array}$ & $\begin{array}{l}\text { Mais le plus souvent il fallait } \\
\text { attendre notre tour. }\end{array}$ \\
\hline 3. & $\begin{array}{l}\text { Dia bertanggung jawab } \\
\text { akan selalu adanya bahan } \\
\text { makanan di rumah. }\end{array}$ & $\begin{array}{l}\text { Elle devait veiller à ce qu'il y ait } \\
\text { toujours des provisions }\end{array}$ \\
\hline 4. & $\begin{array}{l}\text { Bukan karena aku akan pergi } \\
\text { wisma nusantara. }\end{array}$ & $\begin{array}{l}\text { Non pas parce que je devais aller } \\
\text { au WismaNusantara. }\end{array}$ \\
\hline 5. & $\begin{array}{l}\text { Tetapi Anna sudah menguasai } \\
\text { pelajarannya, dan akan ujian } \\
\text { pecan berikutnya. }\end{array}$ & $\begin{array}{l}\text { Mais Anna avait bien révisé et } \\
\text { devait passer son examen la } \\
\text { semaine suivante. }\end{array}$ \\
\hline 6. & $\begin{array}{l}\text { Ku tambahkan agar lain kali } \\
\text { menelepon kebagian } \\
\text { Penerbangan atau dating } \\
\text { untuk membuat janji. }\end{array}$ & $\begin{array}{l}\text { J'ajoutai que la prochaine fois, il } \\
\text { devrait me donner un coup de fil à } \\
\text { la Division des vols ou venir afin } \\
\text { qu'on fixe un rendez-vous. }\end{array}$ \\
\hline
\end{tabular}


Table 4: Apperance list of verb Falloir in French as lexes in Indonesian language in the novel "Telegram" and its equivalence in French in the novel Télégrame

\begin{tabular}{|c|c|c|}
\hline \multirow{2}{*}{ No. } & \multicolumn{2}{|c|}{ FALLOIR } \\
\hline & Indonesia & French \\
\hline 1. & $\begin{array}{l}\text { Seandainya kelakada yang } \\
\text { hendak kau sesalkan, apa } \\
\text { yang akan kau lakukan? }\end{array}$ & $\begin{array}{l}\text { Si tu devais le regretter, plus tard, } \\
\text { que ferais-tu? }\end{array}$ \\
\hline 2. & $\begin{array}{l}\text { Bagaimana kalau kita } \\
\text { berciuman, bergandengan, } \\
\text { bertegur sapa, berpandangan, } \\
\text { hanya karena tugas kelak? }\end{array}$ & $\begin{array}{l}\text { Et si nous devions nous embrasser, } \\
\text { nous tenir pas la main, nous parler, } \\
\text { nous regarder désormais par devoir? }\end{array}$ \\
\hline 3. & $\begin{array}{l}\text { Tetapi sebagai orang yang } \\
\text { masih mempunyai } \\
\text { kesempatan, baiknya } \\
\text { dipikirkan, kalau-kalau ada } \\
\text { jalanlain. }\end{array}$ & $\begin{array}{l}\text { Tant que nous en avions la } \\
\text { possibilité, nous devions réfléchir et } \\
\text { chercher s'il existait une autre voie. }\end{array}$ \\
\hline 4. & $\begin{array}{l}\text { Ada saja kabar-kabar yang } \\
\text { mengganggu ketenangan } \\
\text { orang yang ingin } \\
\text { melangsungkan niat } \\
\text { pribadinya. }\end{array}$ & $\begin{array}{l}\text { Il faut toujours que des nouvelles } \\
\text { viennent troubler la quiétude des gens } \\
\text { plongés dans leurs desseins privés. }\end{array}$ \\
\hline 5. & $\begin{array}{l}\text { Sementara di luar persoalan } \\
\text { itu segalanya berjalan lancer } \\
\text { sebagaimana adatnya. }\end{array}$ & $\begin{array}{l}\text { Mais en dehors de cela, tout se } \\
\text { produisait sans heurt, comme il se } \\
\text { devait. }\end{array}$ \\
\hline 6. & $\begin{array}{l}\text { Tentu dalam hatinya tergores } \\
\text { pula, bahwa ia tidak akan } \\
\text { pernah mempunyai saat-saat } \\
\text { seperti itu. }\end{array}$ & $\begin{array}{l}\text { Elle devait se dire aussi qu'elle ne } \\
\text { connaîtrait jamais d'instant comme } \\
\text { celui-là. }\end{array}$ \\
\hline 7. & $\begin{array}{l}\text { Pasti di rumahnya dia tidak } \\
\text { pernah mendapat perhatian. }\end{array}$ & $\begin{array}{l}\text { Personne ne devait faire attention à } \\
\text { lui, à la maison. }\end{array}$ \\
\hline 8. & $\begin{array}{l}\text { Rupanya aku bener-bener } \\
\text { mabok semalam. }\end{array}$ & Je devais être vraiment ivre, la veille. \\
\hline
\end{tabular}

\subsection{Time, Aspect and Modus}

From the tables above, it is known that time and verb modus contained in falloir does not affect at all the interpretation form of impersonal verb in Indonesian language. The tables showed that interpretation form or verb conjunction does not affect the interpretation at all. In other words, aspect elements implicitly appeared is not explicitly interpretated in Indonesian language. Datas from the four tables showed that verb times determining aspect in sentence either présent, or passé were not explicitly interpretated.

In Indonesian language, the sentence does not use sign particle sudah (perfect tense), telah (perfect tense), sekarang (progressive), akan (future), etc. For example data of number 1 and 2 of the table 1. It is found that meaning of aspect sign did not appear at all in the interpretation. So, particle use of telah or sudah does not has to be appeared.

\subsection{Reasoning Meaning}

Impersonal verb il faut can be equivalent with other verbs that have literal meaning such as devoir. Nevertheless, in particular case, devoir can express a hope (un souhait)or a willingness(un désir)if the interpretation form is in imparfait. As the data number 1 and 2 of 
table 4. The two datas shows that in Indonesian language, there is no harus (must) or its equivalence, but in its interpretation in French found verb devoir which was conjuncted in imparfait form. So, devoir used to express a conditional sentence that express a hope implicitly. This is similir to case number 7 of table 3 where its Indonesian version has advice meaning (le conseil). But in its French version, this meaning was implicitly interpretated through verb interpretation in conditional present.

Next found that impersonal verb meaning il faut can appear on the other verbs which have literal meaning which is different, such as data from number 1, 3, 5, and 7 of table 2. Verbs demeurait, sevrait, ai besoin, tendait have literal meaning which much far from il faut, but its interpretation in Indonesian version has the same meaning, namely harus (must). Hence, this implicit meaning is available in French which is made equivalent with its sentence context.

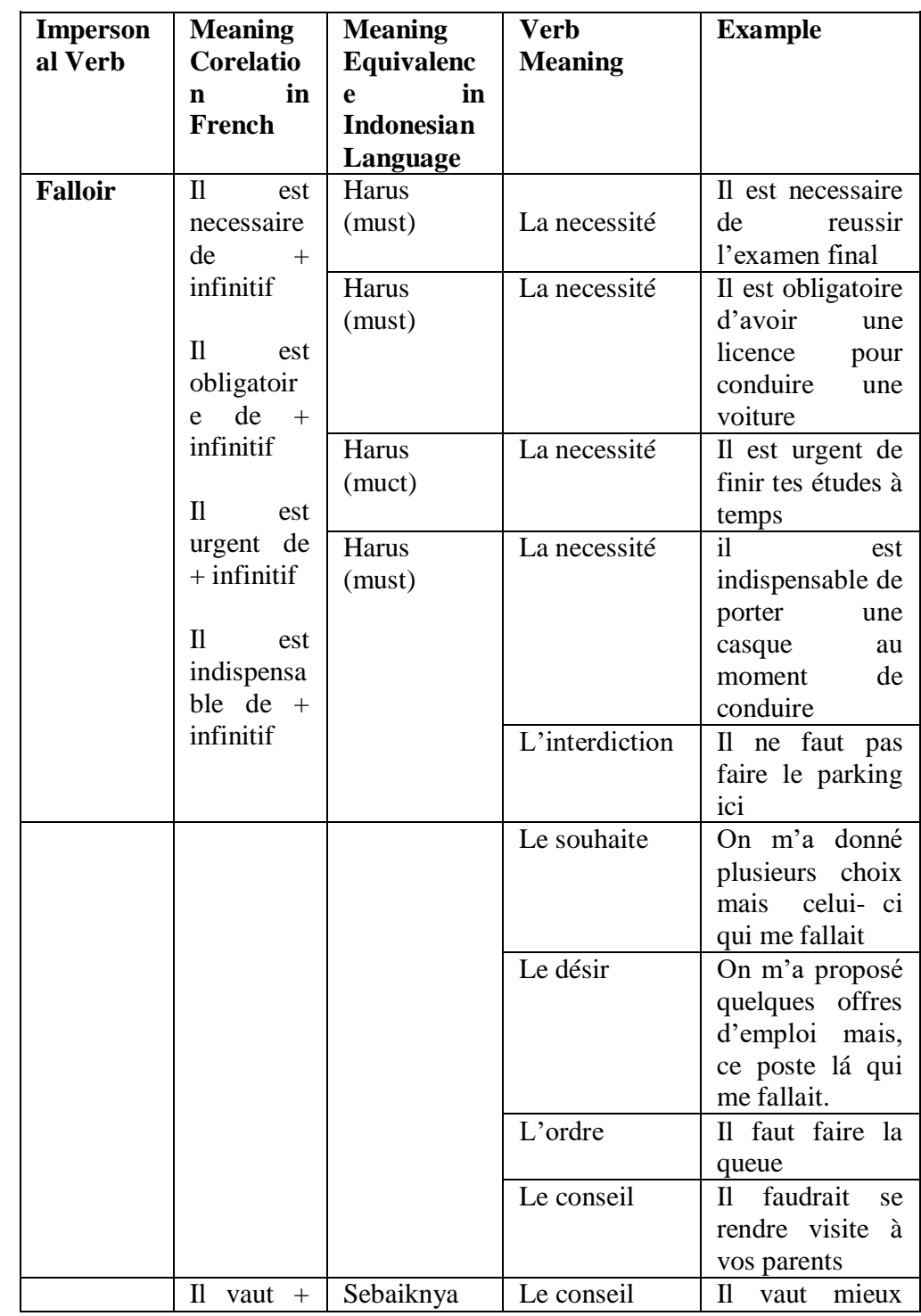




\begin{tabular}{|l|l|l|l|l|}
\hline & infinitif & $\begin{array}{l}\text { (should/had } \\
\text { better })\end{array}$ & $\begin{array}{l}\text { prendre le train } \\
\text { que la voiture }\end{array}$ \\
\hline
\end{tabular}

\subsection{Semi-Auxiliary Verb}

However in other side, it can be seen that particle akan (future tense) which is a sign of undone things can be interpretated with imparfait time, as shown in number 5 and 6 of table 3 . Devais and devait thatshould have past time in its interpretation which has meaning akan (future). Through that, sentence meaning as if happens in the future but in fact the word akan does not mean as the tense sign of the sentence in its interpretation.

Devais which is followed by infinitive alert shows that devais of which literal meaning was equivalent with il faut has function as the semi-auxiliary verb. Due to be followed by infinitive verb, devais has no longer real meaning and this affect the sentence situation.So, this form is called as périphrase verbale. Fleur d'encre (2009) stated that un lien est tissé: comme l'auxiliaire, le semi-auxiliaire présente un sens différent de son sens habituel.

The same with data number 8 of table 2 that has avoir in the form of conditionnel present. The function of avoir is also a semi-auxiliary verb which has no full meaning 'have'. In this sentence, avoir that functions as modal verb gives meaning and situation like "obligation" (l'obligation).

\section{Conclusion}

After making detail observation about the use of impersonal verb from tables made, it is found that il faut of which literal meaning is harus (obligation) can implicitly appear in French and explicitly in Indonesian language or conversely. Then, il faut that has literal meaning in French may be equivalent with other verbs that have no corelation with il faut, but interpretated into its equivalence in Indonesian language. This equivalence process is made based context and sentence reasoning through 3 things that are always overlapped, namely tenses, aspect, and modus in French.

\section{References}

[1]Caroline Lachet, "Des savoirs scientifiques aux savoirs scolaires : entre élaboration des connaissances et élaboration du discours. Application à l'aspect verbal », Lidil [En ligne], $47 \mid 2013$, mis en ligne le 01 décembre 2014, consulté le 15 mai 2018. URL : http://journals.openedition.org/lidil/3267

[2]Chollet, Isabelle et Robert, Jean Michel. 2009. Précis de Grammaire. Espagne: CLE International

[3]Fleur d'encre 3e. Paris : Hachette, (2012)

[4] Hoed, Benny Hoendoro.: Liberté en Traduction, Skopos, et Idéologie : Finalité et Communication Interculturelle, in Bandung : Hôtel Savoy Homann (éds), Actes du Colloque International, La Francophonie dans Les Pays Non Francophones, 11-12 (2004)

[5] Samsuri.: Analisis Bahasa. Jakarta : Erlangga (1983)

[6] Sudaryanto.: Metode dan Aneka Teknik Analisis Bahasa. Yogyakarta : Duta Wacana University.(1993) 